\title{
A STOCHASTIC MAXIMIN FIXED-POINT EQUATION RELATED TO GAME TREE EVALUATION
}

\author{
GEROLD ALSMEYER *** AND \\ MATTHIAS MEINERS, ${ }^{*}$ Westfälische Wilhelms-Universität Münster
}

\begin{abstract}
After suitable normalization the asymptotic root value $W$ of a minimax game tree of order $b \geq 2$ with independent and identically distributed input values having a continuous, strictly increasing distribution function on a subinterval of $\mathbb{R}$ appears to be a particular solution of the stochastic maximin fixed-point equation $W \stackrel{\mathscr{L}}{=} \xi \max _{1 \leq i \leq b} \min _{1 \leq j \leq b} W_{i, j}$, where $W_{i, j}$ are independent copies of $W$ and $\stackrel{\mathcal{L}}{=}$ denotes equality in law. Moreover, $\xi=g^{\prime}(\alpha)>1$, where $g(x):=\left(1-(1-x)^{b}\right)^{b}$ and $\alpha$ denotes the unique fixed point of $g$ in $(0,1)$. This equation, which takes the form $F(t)=g(F(t / \xi))$ in terms of the distribution function $F$ of $W$, is studied in the present paper for a reasonably extended class of functions $g$ so as to encompass more general stochastic maximin equations as well. A complete description of the set of solutions $F$ is provided followed by a discussion of additional properties such as continuity, differentiability, or existence of moments. Based on these results, it is further shown that the particular solution mentioned above stands out among all other ones in that its distribution function is the restriction of an entire function to the real line. This extends recent work of Ali Khan, Devroye and Neininger (2005). A connection with another class of stochastic fixed-point equations for weighted minima and maxima is also discussed.
\end{abstract}

Keywords: Minimax game tree; stochastic fixed point; maximin equation; moments; weighted minima; weighted maxima

2000 Mathematics Subject Classification: Primary 60E05

$$
\text { Secondary 68Q25 }
$$

\section{Introduction}

In complex two-person full information games like chess and go, players can typically search for best moves only a finite number of steps ahead. The possible moves and the terminal positions under this constraint can be represented in a rooted, so called minimax tree with fixed branching degree $b \geq 2$ and height $2 k, k \geq 0$, where $b$ gives the number of possible moves per turn of a player and $2 k$ is the searching horizon. The terminal positions are represented by the leaves of the tree which are carrying random values $V_{1}, \ldots, V_{n}, n=b^{2 k}$, where large values indicate a position that favors player one while smaller values favor player two. All other nodes of the tree are labeled with $\wedge$ (minimum) on odd levels and with $\vee$ (maximum) on even levels. The value of a node can be evaluated by applying its labeling operator to the values of the children. This corresponds to player one always choosing the move with maximal value whereas player two always chooses the move with minimal value. In the present paper

\footnotetext{
Received 12 February 2007.

* Postal address: Institut für Mathematische Statistik, Fachbereich Mathematik und Informatik, Westfälische WilhelmsUniversität Münster, Einsteinstrasse 62, D-48149 Münster, Germany.

** Email address: gerolda@math.uni-muenster.de
} 
we are concerned with the asymptotic root value $Z_{k}$ of such a minimax tree as $k$ tends to $\infty$ and assuming that $V_{1}, \ldots, V_{n}$ are independent and identically distributed (i.i.d.). This problem was first studied by Pearl [7], who showed that $Z_{k}$, without any scaling, converges in probability to a constant $c$,

$$
Z_{k} \stackrel{\mathrm{P}}{\rightarrow} c \quad \text { as } k \rightarrow \infty
$$

under the condition that the common distribution function $F_{V}$, say, of the $V_{j}$ is continuous and strictly increasing on $\left\{0<F_{V}<1\right\}$. He gave a further characterization of $c$, namely

$$
c=F_{V}^{-1}(\alpha),
$$

with $\alpha$ denoting the unique fixed point in $(0,1)$ of the function

$$
g(x):=\left(1-(1-x)^{b}\right)^{b} .
$$

The result was recently strengthened by Ali Khan et al. [2, Theorem 1] who showed that, with $\xi:=g^{\prime}(\alpha)>1$,

$$
\xi^{k}\left(F_{V}\left(Z_{k}\right)-\alpha\right) \stackrel{\mathcal{L}}{\rightarrow} W^{*} \text { as } k \rightarrow \infty
$$

where $\stackrel{\mathcal{L}}{\rightarrow}$ denotes convergence in law. Moreover, the distribution $\mathcal{L}\left(W^{*}\right)$ of $W^{*}$, which does not depend on the distribution $\mathscr{L}(V)$ of the leaf weights $V_{1}, \ldots, V_{n}$, has a continuous distribution function $F^{*}$ with $0<F^{*}<1, F^{*}(0)=\alpha$, and

$$
F^{*}(x)=g\left(F^{*}\left(\frac{x}{\xi}\right)\right), \quad x \in \mathbb{R},
$$

where $g$ is as defined in (1.1). The invariance of $\mathcal{L}\left(W^{*}\right)$ becomes quite obvious when observing that $F_{V}\left(Z_{k}\right)-\alpha$ is nothing but the root value of the tree when replacing $V_{1}, \ldots, V_{n}$ with $F_{V}\left(V_{1}\right)-\alpha, \ldots, F_{V}\left(V_{n}\right)-\alpha$ which, besides again being i.i.d., are always having a uniform distribution on $(-\alpha, 1-\alpha)$. The above result could therefore be restated as follows.

Theorem 1.1. Let $g$ be given by (1.1) for some $b \geq 2$ and $V_{1}, V_{2}, \ldots$ be i.i.d. random variables with a uniform distribution on $(-\alpha, 1-\alpha)$, where $\alpha$ denotes the unique fixed point of $g$ in $(0,1)$. Then the asymptotic root value $Z_{k}$, as defined above, satisfies

$$
\xi^{k} Z_{k} \stackrel{\mathcal{L}}{\rightarrow} W^{*} \text { as } k \rightarrow \infty
$$

where $\xi=g^{\prime}(\alpha)$ and $W^{*}$ has a continuous distribution function $F^{*}$ satisfying $0<F^{*}<1$, $F^{*}(0)=\alpha$, and (1.3).

Equation (1.3) means that $W^{*}$ (more precisely its law $\mathcal{L}\left(W^{*}\right)$ ) is a solution of the stochastic fixed-point equation

$$
W \stackrel{\mathscr{L}}{=} \xi \max _{1 \leq i \leq b} \min _{1 \leq j \leq b} W_{i, j}
$$

where the $W_{i, j}$ are independent copies of $W$. Involving maxima and minima simultaneously, this equation is similar and yet different from the max-type fixed-point equations discussed extensively in a recent survey by Aldous and Bandyopadhyay [1] and also in [6] and [4]. On the other hand, there is a connection to a pure max-type equation studied by Alsmeyer and Rösler [3], namely to

$$
W \stackrel{\mathcal{L}}{=} \max _{1 \leq j \leq b} T_{j} W_{j}
$$




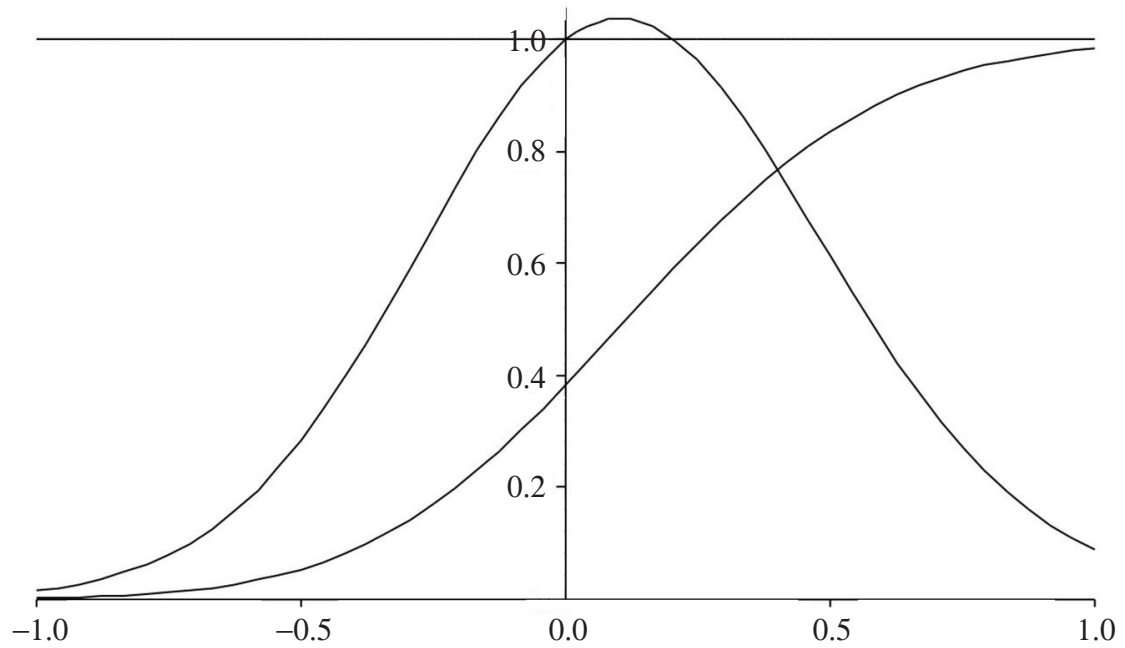

FIGURE 1: An approximation of $F^{*}$ and its derivative (density function) based on $a_{0}, \ldots, a_{50}$ recursively computed using (1.6). This plot may be compared to a similar one in [2] obtained by using the fixed point equation (1.3).

in the case where the $T_{j}$ are negative constants. Indeed, if $T_{1}=\cdots=T_{b}$ and $\xi:=T_{1}^{2}$, then (1.5) takes the form of (1.4) after one iteration. Further information will be given in Subsection 4.3.

Neininger (personal communication) raised the question of the uniqueness of the distribution of $W^{*}$ as a solution to (1.4) (modulo scaling by positive factors) and thus of a description of all solutions. The latter is the main purpose of this article. We will show that, in fact, there exists a whole continuum of solutions to (1.4) among which the limit law $\mathcal{L}\left(W^{*}\right)$ in (1.2) stands out by having the following particular smoothness properties.

Theorem 1.2. Under the stated conditions, the distribution function $F^{*}$ is the restriction of an entire function to the real line and, thus, may be written as an everywhere convergent power series $F^{*}(x)=\sum_{n \geq 0} a_{n} x^{n}$. In particular, $F^{*}$ is infinitely often differentiable and the unique solution to (1.3) with the property that $F^{*}(0)=\alpha$ and $F^{*^{\prime}}(0)=1$. The coefficients $a_{n}, n \geq 0$, of the above expansion satisfy $a_{0}=\alpha, a_{1}=1$, and

$$
a_{n}=\frac{1}{\xi^{n}-\xi} \sum_{k=2}^{b^{2}} c_{k} \sum_{\substack{j_{1}+\ldots+j_{k}=n, j_{1} \geq 1, \ldots, j_{k} \geq 1}} a_{j_{1}} \cdots a_{j_{k}}
$$

for $n \geq 2$, where $c_{0}, \ldots, c_{b^{2}}$ are the coefficients of $g$ in its polynomial expansion about $\alpha$. Moreover, the moment-generating function $\Psi_{F^{*}}(t):=\int \mathrm{e}^{t x} F^{*}(\mathrm{~d} x)$ of $F^{*}$ is finite for all $t \in \mathbb{R}$.

A plot of $F^{*}$ and its derivative, obtained by recursive calculation of $a_{2}, \ldots, a_{50}$ using (1.6), is shown in Figure 1. We will prove Theorem 1.2 in Section 6 and finish our introduction with a short outline of the rest of this article. In Section 2 we discuss a more general version of the fixed-point equation (1.4). More precisely, its equivalent version (1.3) will be studied for a fairly general class of functions $g$. The main results provide us with a complete description of the set of solutions to this equation and conditions that ensure existence of continuous or even 
differentiable solutions when viewed as distribution functions (notationally, no distinction is made throughout this article between a distribution and its distribution function). In Section 3 we focus on the question of existence of moments of solutions. We return to maximin fixedpoint equations including (1.4) in Section 4 by presenting a discussion of it in light of the previously obtained results, examining the case in which the numbers of occurring maxima and minima are random variables, and analyzing its connection with another fixed-point equation for weighted maxima and minima that has been studied in [3]. All proofs are given in Sections 5 and 6 followed by concluding remarks in Section 7. An appendix collects some basic properties of the functions $g$ considered in (1.3).

\section{The general fixed-point equation and its solutions}

Returning to (1.4) let us note that it does not much complicate the situation to consider the following more general equation:

$$
W \stackrel{\mathcal{L}}{=} \xi \max _{1 \leq i \leq n} \min _{1 \leq j \leq m_{i}} W_{i, j}
$$

with integers $m_{1}, \ldots, m_{n}, n \geq 2$. Expressing (2.1) in terms of distribution functions (which we do not distinguish notationally from the pertinent distribution), we have (compare with (1.3))

$$
F(t)=\prod_{i=1}^{n}\left(1-\left(1-F\left(\frac{t}{\xi}\right)\right)^{m_{i}}\right)=g \circ F\left(\frac{t}{\xi}\right)
$$

for all $t \in \mathbb{R}$, where $g(x)=\prod_{i=1}^{n}\left(1-(1-x)^{m_{i}}\right)$ is a polynomial and an element from the class $g$, say, of continuously differentiable, strictly increasing functions $g:[0,1] \rightarrow[0,1]$ satisfying $g(c)=c$ for $c \in\{0,1\}$ and finitely many, but at least one $c \in(0,1)$ (for the former polynomial this $c$ is in fact unique, see Lemma A.2 in the appendix). Since the class $g$ is actually an appropriate function class for our task, we will first study (2.2) for general functions $g \in g$ and $\xi>1$. A discussion of the maximin situation with polynomial $g$ as stated above is postponed until Section 4. Denote by $\mathfrak{F}_{g, \xi}$ the set of all solutions of (2.2) and note that this set always contains the trivial solution $\delta_{0}$, the Dirac measure at 0 . Let $\mathfrak{F}_{g, \xi}^{+}$and $\mathfrak{F}_{g, \xi}^{-}$be the subsets of fixed points having mass 0 on the negative and positive halfline, respectively.

With the stated extension of the function $g$, further fixed-point equations are covered as well, for instance,

$$
W \stackrel{\mathscr{L}}{=} \xi \max _{1 \leq i \leq n} \min _{1 \leq j \leq m_{i}} \max _{1 \leq k \leq n_{i j}} \min _{1 \leq l \leq m_{i j k}} W_{i, j, k, l}
$$

where again $g$ is a polynomial, or (2.2) with random numbers $n, m_{1}, \ldots, m_{n}$, in which case $g \in g$ is generally not a polynomial (see Section 4.2 for further details).

Let $\mathcal{L}_{g}$ denote the finite set of fixed points of $g$, i.e. $\mathcal{L}_{g}:=\{x \in[0,1]: g(x)=x\} \supset\{0,1\}$. The relevance of the fixed points of $g$ for our purposes becomes apparent when observing that, by (2.2) and the continuity of $g$, any $F \in \mathfrak{F}_{g, \xi}$ must satisfy

$$
F(0-)=g(F(0-)) \quad \text { and } \quad F(0)=g(F(0)),
$$

where $F(x-):=\lim _{y \uparrow x} F(y)$. Define

$$
c_{0}:=\inf \mathcal{L}_{g} \backslash\{0\} \quad \text { and } \quad c_{1}:=\sup \mathcal{L}_{g} \backslash\{1\},
$$


Case (i)

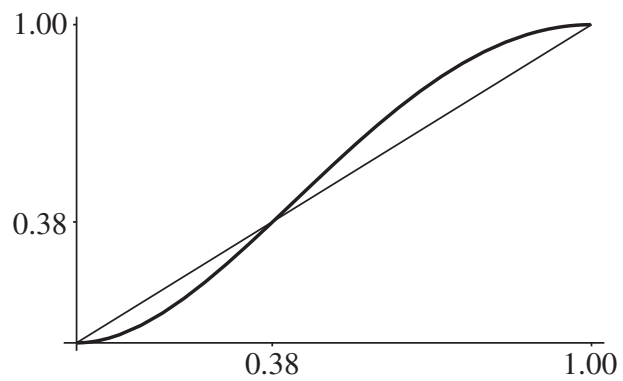

Case (iii)

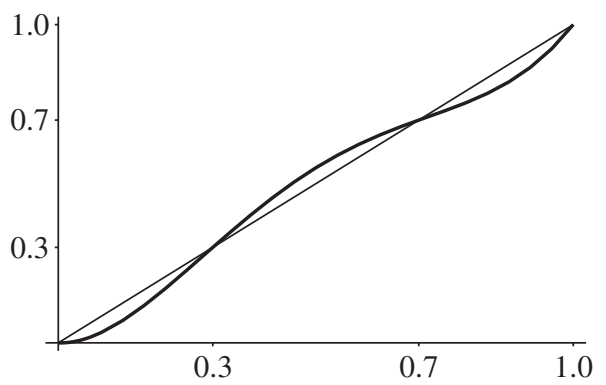

Case (ii)

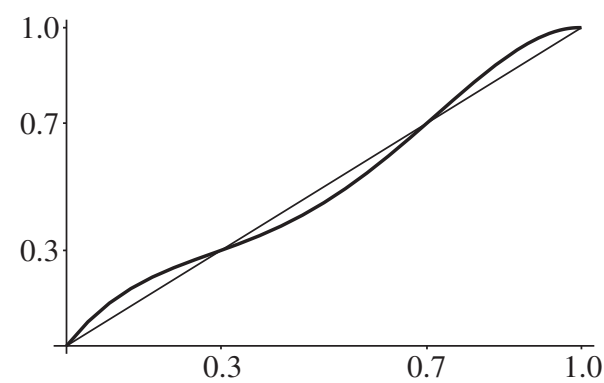

Case (iv)

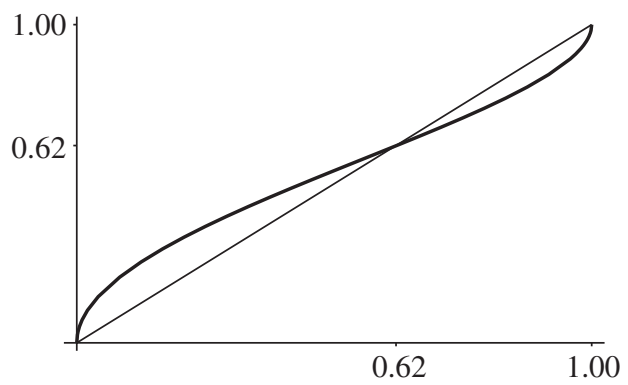

FIGURE 2: Examples illustrating cases (i)-(iv).

which are both (possibly equal) elements of $\mathcal{L}_{g}$. Furthermore, $0<c_{0} \leq c_{1}<1$, as $g$ has at least one fixed point in $(0,1)$. With a little more effort we can verify that $F(0-) \in\left\{0, c_{0}\right\}$ and $F(0) \in\left\{c_{1}, 1\right\}$ (see Lemma 5.1). The following four cases will be distinguished hereafter so as to state our results in a comprehensive way.

Case (i): $g(x)<x$ for $0<x<c_{0}$ and $g(x)>x$ for $c_{1}<x<1$.

Case (ii): $g(x)>x$ for $0<x<c_{0}$ and $g(x)>x$ for $c_{1}<x<1$.

Case (iii): $g(x)<x$ for $0<x<c_{0}$ and $g(x)<x$ for $c_{1}<x<1$.

Case (iv): $g(x)>x$ for $0<x<c_{0}$ and $g(x)<x$ for $c_{1}<x<1$.

Figure 2 shows a possible shape of $g$ for each of these cases.

We further need the following function classes. Fixing any $s_{0}, t_{0} \in \mathbb{R}, s_{0}<0<t_{0}$, let $\mathcal{F}_{-}$ and $\mathcal{F}_{+}$be the classes of nondecreasing, right-continuous functions $f_{-}:\left[\xi s_{0}, s_{0}\right) \rightarrow[0, \infty)$ and $f_{+}:\left[t_{0}, \xi t_{0}\right) \rightarrow[0, \infty)$, respectively, satisfying $0 \leq f_{-}\left(\xi s_{0}\right)<c_{0}, c_{1}<f_{+}\left(t_{0}\right) \leq 1$,

$$
f_{-}(s) \leq g^{-1}\left(f_{-}\left(\xi s_{0}\right)\right) \text { for } s \in\left[\xi s_{0}, s_{0}\right),
$$

and

$$
f_{+}(t) \leq g\left(f_{+}\left(t_{0}\right)\right) \quad \text { for } t \in\left[t_{0}, \xi t_{0}\right) .
$$

Here $g^{-1}$ denotes the inverse function of $g$. We also write $g^{n}$ for $|n|$-fold composition of $g$ $(n \geq 1)$, or of its inverse $g^{-1}(n \leq-1)$, and let $g^{0}$ be the identity function. 
Theorem 2.1. Let $g \in g$ and $\xi>1$. Arbitrarily fixing $s_{0}, t_{0} \in \mathbb{R}, s_{0}<0<t_{0}$, the set $\mathfrak{F}_{g, \xi}$ can be described as follows.

(a) (Case (i).) There is a one-to-one correspondence between $\mathfrak{F}_{g, \xi}$ and $\mathcal{F}_{-} \times \mathcal{F}_{+}$in the sense that any $F \in \mathfrak{F}_{g, \xi}$ is of the form

$$
F(t)= \begin{cases}g^{n}\left(f_{+}\left(\frac{t}{\xi^{n}}\right)\right) & \text { if } t>0 \\ g^{n}\left(f_{-}\left(\frac{t}{\xi^{n}}\right)\right) & \text { if } t<0, \\ c_{1} & \text { if } t=0 \text { and } f_{+}\left(t_{0}\right)<1, \\ 1 & \text { if } t=0 \text { and } f_{+}\left(t_{0}\right)=1,\end{cases}
$$

for some pair $\left(f_{-}, f_{+}\right) \in \mathcal{F}_{-} \times \mathcal{F}_{+}$, where $n \in \mathbb{Z}$ is the unique integer satisfying $t_{0} \leq t / \xi^{n}<\xi t_{0}$ if $t>0$, and $\xi s_{0} \leq t / \xi^{n}<s_{0}$ if $t<0$.

(b) (Case (ii).) There is a one-to-one correspondence between $\mathfrak{F}_{g, \xi}$ and $\mathcal{F}_{+}$in the sense that any $F \in \mathfrak{F}_{g, \xi}$ is of the form of (2.4) for some $f_{+} \in \mathcal{F}_{+}$and with $f_{-} \equiv 0$. In particular, any such $F$ is concentrated on the nonnegative halfine, i.e. $\mathfrak{F}_{g, \xi}=\mathfrak{F}_{g, \xi}^{+}$.

(c) (Case (iii).) There is a one-to-one correspondence between $\mathfrak{F}_{g, \xi}$ and $\mathcal{F}_{-}$in the sense that any $F \in \mathfrak{F}_{g, \xi}$ is of the form of (2.4) for some $f_{-} \in \mathcal{F}_{-}$and with $f_{+} \equiv 1$. In particular, any such $F$ is concentrated on the nonpositive halfline, i.e. $\mathfrak{F}_{g, \xi}=\mathfrak{F}_{g, \xi}^{-}$.

(d) (Case (iv).) There is no nontrivial solution, i.e. $\mathfrak{F}_{g, \xi}=\left\{\delta_{0}\right\}$.

Thus, we conclude that every nontrivial solution $F \in \mathfrak{F}_{g, \xi}$, once being defined locally on the intervals $\left[\xi s_{0}, s_{0}\right) \subset(-\infty, 0)$ and/or $\left[t_{0}, \xi t_{0}\right) \subset(0, \infty)$ for arbitrary $s_{0}<0<t_{0}$, is then already determined everywhere with the possible restriction that it be concentrated on one of the halflines. Also, since $\mathcal{F}_{-}$and $\mathcal{F}_{+}$contain $f_{-} \equiv 0$ and $f_{+} \equiv 1$, respectively, the fixed-point property persists whenever a two-sided $F$ (as a probability measure) is restricted to one of the halflines. Consequently, the one-to-one correspondence stated in Theorem 2.1(a) for case (i) may also be viewed as a one-to-one correspondence between $\mathfrak{F}_{g, \xi}$ and $\mathfrak{F}_{g, \xi}^{-} \times \mathfrak{F}_{g, \xi}^{+}$.

Our next result shows that continuity or even differentiability of $F \in \mathfrak{F}_{g, \xi}$ on $\mathbb{R} \backslash\{0\}$ follow when putting further restrictions on $f_{-}$and $f_{+}$.

Theorem 2.2. In the situation of Theorem 2.2, a solution $F \in \mathfrak{F}_{g, \xi}$, defined by (2.4), satisfies the following.

(a) $F$ is continuous on $\mathbb{R} \backslash\{0\}$, if $f_{-}$and $f_{+}$are continuous with $f_{-}\left(\xi s_{0}\right)=g\left(f_{-}\left(s_{0}\right)\right)$, $f_{+}\left(\xi t_{0}\right)=g\left(f_{+}\left(t_{0}\right)\right)$

$$
f_{-}\left(s_{0}\right) \begin{cases}\in\left[0, c_{0}\right) & \text { if } g(x)<x \text { for } x \in\left(0, c_{0}\right), \\ =0 & \text { otherwise }\end{cases}
$$

and

$$
f_{+}\left(t_{0}\right) \begin{cases}\in\left(c_{1}, 1\right] & \text { if } g(x)>x \text { for } x \in\left(c_{1}, 1\right), \\ =1 & \text { otherwise. }\end{cases}
$$

It is also continuous at 0 if $f_{-}\left(s_{0}\right)>0, f_{+}\left(t_{0}\right)<1$, and $g$ has exactly one fixed point in $(0,1)$. 
(b) $F$ is differentiable on $\mathbb{R} \backslash\{0\}$, if $g, f_{-}$, and $f_{+}$are differentiable on $[0,1]$, $\left[\xi s_{0}, s_{0}\right]$, and $\left[t_{0}, \xi t_{0}\right]$, respectively (in the one-sided sense at the boundaries), with $g^{\prime}>0$ on $\left(0, c_{0}\right) \cup\left(c_{1}, 1\right), f_{-}^{\prime}\left(s_{0}\right)=\xi^{-1} g^{\prime}\left(f_{-}\left(s_{0}\right)\right) f_{-}^{\prime}\left(s_{0}\right)$, and $f_{+}^{\prime}\left(\xi t_{0}\right)=\xi^{-1} g^{\prime}\left(f_{+}\left(t_{0}\right)\right) f_{+}^{\prime}\left(t_{0}\right)$.

The question of differentiability of $F$ at 0 is more delicate and cannot be generally answered. We return to this point in Section 6 in connection with the proof of Theorem 1.2.

Our last result of this section follows, essentially, from the previous one and provides the necessary and sufficient conditions that ensure the existence of a continuous solution $F \in \mathfrak{F}_{g, \xi}$.

Corollary 2.1. Given any $\xi>1$ and $g \in \mathcal{g}$, the fixed-point equation (2.2) has a continuous solution if, and only if, $g$ has exactly one fixed point $\alpha \in(0,1)$ and $g(x) \gtrless x$ for $x \gtrless \alpha$, $x \in(0,1)$.

All results of this section will be proved in Section 5 .

\section{The existence of moments}

The following two results will be concerned with the existence of moments for solutions $F \in \mathfrak{F}_{g, \xi}$ which is most efficiently done by studying $F$ on $(0, \infty)$ and $(-\infty, 0)$ separately. Recalling the remarks after Theorem 2.2 it is in fact sufficient to study the moments of the elements of $\mathfrak{F}_{g, \xi}^{-}$and $\mathfrak{F}_{g, \xi}^{+}$. Therefore, for $p>0$, let $\mathbb{M}_{p}^{+}$and $\mathbb{M}_{p}^{-}$denote the sets of distributions $F$ having a finite $p$ th moment on $(0, \infty)$ and $(-\infty, 0)$, respectively, i.e.

$$
\mathbb{M}_{p}^{+}:=\left\{F: \int_{(0, \infty)} x^{p} F(\mathrm{~d} x)<\infty\right\}
$$

and similarly for $\mathbb{M}_{p}^{-}$. Also, let $\mathbb{M}_{<p}^{ \pm}:=\bigcap_{q<p} \mathbb{M}_{q}^{ \pm}$be the set of distributions with finite moments of order $q<p$ on the associated halflines, and set $\mathbb{M}_{\infty}^{ \pm}=\bigcap_{p>0} \mathbb{M}_{p}^{ \pm}$.

Theorem 3.1. Given $\xi>1$ and $g \in \mathcal{G}$, set

$$
p_{0}:=\frac{\log \left(1 / g^{\prime}(0)\right)}{\log \xi} \text { and } p_{1}:=\frac{\log \left(1 / g^{\prime}(1)\right)}{\log \xi},
$$

which are interpreted in the usual way as $\infty$ if $g^{\prime}(0)=0$ and $g^{\prime}(1)=0$, respectively. Then the following assertions hold true.

(a) If $p_{0}>0$, then $\mathfrak{F}_{g, \xi}^{-} \neq \varnothing$ and $\mathfrak{F}_{g, \xi}^{-} \subset \mathbb{M}_{<p_{0}}^{-}$. In particular, $\mathfrak{F}_{g, \xi}^{-} \subset \mathbb{M}_{\infty}^{-}$in the case in which $p_{0}=\infty$.

(b) If $p_{0}<\infty$, then $\mathfrak{F}_{g, \xi}^{-} \cap \mathbb{M}_{p}=\varnothing$ for any $p>p_{0}$.

(c) If $p_{1}>0$, then $\mathfrak{F}_{g, \xi}^{+} \neq \varnothing$ and $\mathfrak{F}_{g, \xi}^{+} \subset \mathbb{M}_{<p_{1}}^{+}$. In particular, $\mathfrak{F}_{g, \xi}^{+} \subset \mathbb{M}_{\infty}^{+}$in the case in which $p_{1}=\infty$.

(d) If $p_{1}<\infty$, then $\mathfrak{F}_{g, \xi}^{+} \cap \mathbb{M}_{p}=\varnothing$ for any $p>p_{1}$.

Thus, we conclude that the existence of (polynomial) moments is essentially tied to the behavior of $g^{\prime}$ at 0 and 1 . For two-sided fixed points $F$, which only exist in case (i), Theorem 3.1 directly implies that $\int|x|^{p} F(\mathrm{~d} x)$ is finite for all $p<\min \left(p_{0}, p_{1}\right)$ and infinite for $p>$ $\min \left(p_{0}, p_{1}\right)$. Another immediate consequence of the result is that exponential moments of any $F \neq \delta_{0}$ can only exist if at least one of $g^{\prime}(0)$ and $g^{\prime}(1)$ equals 0 . Assuming the latter, our next result provides conditions which are either necessary or sufficient for the 
existence of an exponential moment of the order $s \in \mathbb{R}$ for $F \in \mathfrak{F}_{g, \xi}$, that is of $\Psi_{F}(s)$, where $\Psi_{F}(t):=\int \mathrm{e}^{t x} F(\mathrm{~d} x)$ is the moment-generating function (MGF) of $F$.

Proposition 3.1. Given $\xi>1, g \in g$, and any $F \in \mathfrak{F}_{g, \xi}$, set $c_{k}^{+}:=1-g^{k}(F(1))$ and $c_{k}^{-}:=g^{k}(F(-1))$, for $k \geq 0$. Then

$$
\sum_{k \geq 0} \mathrm{e}^{ \pm s \xi^{k+1}} c_{k}^{ \pm}<\infty \Longrightarrow \Psi_{F}( \pm s)<\infty \Longrightarrow \sum_{k \geq 1} \mathrm{e}^{ \pm s \xi^{k}} c_{k}^{ \pm}<\infty
$$

holds true for each $s>0$.

Thus, we see that the existence of $\Psi_{F}(s)$ for any $s \neq 0$ depends very sensitively on the rate at which $g^{k}(F(-1))$ or $1-g^{k}(F(1))$ approaches 0 as $k \rightarrow \infty$. The relevance of both quantities for the tail behavior of $F$ becomes apparent when observing that, by (2.2),

$$
g^{k}(F(-1))=F\left(-\xi^{k}\right) \text { and } g^{k}(F(1))=F\left(\xi^{k}\right),
$$

for each $k \geq 0$. Naturally, this will enter into the proof. More transparent conditions on the finiteness of $\Psi_{F}(s)$ will be provided in the next section for the general maximin fixed-point equation (2.1).

\section{Maximin fixed-point equations revisited}

\subsection{The general maximin fixed-point equation}

Returning to (2.1), that is to

$$
W \stackrel{\mathcal{L}}{=} \xi \max _{1 \leq i \leq n} \min _{1 \leq j \leq m_{i}} W_{i, j}
$$

with $\xi>1$ and integers $n, m_{1}, \ldots, m_{n} \geq 2$, leads us to a study of (2.2) for polynomials $g$ of the form

$$
g(x)=\prod_{i=1}^{n}\left(1-(1-x)^{m_{i}}\right) .
$$

It is shown in Lemma A.2 of the appendix that $g$ has exactly one fixed point $c$ in $(0,1)$, so $c_{0}=c_{1}=c$, and that $g^{\prime}(0)=g^{\prime}(1)=0$ (implying case (i)). By Theorem 3.1, the last fact ensures that every solution has moments of any polynomial order and suggests looking at exponential moments or, in other words, looking at the MGF, $\Psi_{F}(s)$, of a solution $F \in \mathfrak{F}_{g, \xi}$. As disclosed by Proposition 3.1, finiteness of $\Psi_{F}(s)$ for $s \neq 0$ depends on the rate of decay of one of the sequences

$$
1-g^{n}(F(1))=1-F\left(\xi^{n}\right) \quad \text { or } \quad g^{n}(F(-1))=F\left(-\xi^{n}\right), \quad n \geq 0,
$$

but further information can be given for the situation here.

Theorem 4.1. Under the previous assumptions set $m:=\min \left(m_{1}, \ldots, m_{n}\right)$ and let $F \in \mathfrak{F}_{g, \xi}$. Then the following assertions hold true.

(a) The MGF, $\Psi_{F}(s)$, is finite for all $s>0$ if $\xi<m$, and is in a right neighborhood of 0 if $\xi=m$.

(b) If $F$ has positive mass on $(0, \infty)$ and $\xi>m$, then $\Psi_{F}(s)=\infty$ for each $s>0$. 
(c) The MGF, $\Psi_{F}(s)$, is finite for all $s<0$ if $\xi<n$, and in a left neighborhood of 0 if $\xi=n$.

(d) If $F$ has positive mass on $(-\infty, 0)$ and $\xi>n$, then $\Psi_{F}(s)=\infty$ for each $s<0$.

The proof of this result will be given in Section 5 after the proof of Proposition 3.1, on which it is based.

\subsection{The maximin fixed-point equation involving random numbers of maxima and minima}

Replacing $n, m_{1}, \ldots, m_{n}$ with integer-valued random variables $N, M_{1}, \ldots, M_{N}$ in (2.1) leads to the fixed-point equation

$$
W \stackrel{\mathcal{L}}{=} \xi \max _{1 \leq i \leq N} \min _{1 \leq j \leq M_{i}} W_{i, j},
$$

where the $W_{i, j}$ are independent copies of $W$ which are also independent of $\left(N, M_{1}, \ldots, M_{N}\right)$. Suppose that $N, M_{1}, M_{2} \ldots$ are positive and independent and, furthermore, that $M_{1}, M_{2}, \ldots$ are identically distributed with generic copy $M$. Let $f_{N}(s)=\mathrm{E} s^{N}$ and $f_{M}(s)=\mathrm{E} s^{M}$ denote the generating functions of $N$ and $M$, respectively. A simple calculation shows that under these assumptions (4.1), in terms of distribution functions, again takes the form $F(t)=g \circ F(t / \xi)$, but with $g(x)=f_{N}\left(1-f_{M}(1-x)\right.$ ). Plainly, $g(0)=0$ and $g(1)=1$, but $g$ does not need to be an element of $g$, in that it may have infinitely many fixed points in $(0,1)$ (see Example 4.3, below) or may fail to be continuously differentiable at 0 or 1 (for instance, if E $M=\infty$ and $\mathrm{P}(N=1)>0)$. The following lemma provides sufficient conditions for $\mathcal{L}_{g}$ to be finite.

Lemma 4.1. Each of the following two conditions is sufficient for $\mathcal{L}_{g}$ to be finite:

(C1) both $\mathrm{P}(N=1) \mathrm{E} M$ and $\mathrm{P}(M=1) \mathrm{E} N$ are not equal to 1 ,

(C2) $f_{N}(x)<\infty$ and $f_{M}(x)<\infty$ for some $x>1$, and $g(x) \not \equiv x$.

Proof. Suppose (C1) holds. Since both $g^{\prime}(0)=\mathrm{P}(N=1)$ E $M$ and $g^{\prime}(1)=\mathrm{P}(M=1)$ E $N$ are not equal to 1 , we must have $\mathcal{L}_{g} \backslash\{0,1\} \subset[\varepsilon, 1-\varepsilon]$ for some $\varepsilon>0$. Now, if $\mathscr{L}_{g}$ is infinite it contains an accumulation point in the compact set $[\varepsilon, 1-\varepsilon]$ and thus in $(0,1)$, which in turn leads to the impossible conclusion that $g(x)=x$ on $[0,1]$ as $g$ is analytic on $(0,1)$.

Assuming (C2), the function $g$ is analytic in some $(-\varepsilon, 1+\varepsilon), \varepsilon>0$. Hence, if $\mathcal{L}_{g}$ is infinite it has an accumulation point in this interval implying that $g(x) \equiv x$. But this is excluded by assumption.

Clearly, under condition (C1) or (C2) the set of solutions to (4.1) is completely described by Theorem 2.1. We close this subsection with an example that shows that $g(x) \equiv x$ may occur even if $N$ and $M$ have nondegenerate distributions on $\mathbb{N}$.

Example 4.1. Suppose that $f_{N}(x)=2^{x}-1$ and $f_{M}(x)=1-(\log 2)^{-1} \log (2-x)$; thus,

$$
\mathrm{P}(N=n)=\frac{(\log 2)^{n}}{n !} \quad \text { and } \quad \mathrm{P}(M=n)=\frac{1}{\log 2} \frac{1}{n 2^{n}},
$$

for $n \in \mathbb{N}$. It is then readily verified that $g(x)=f_{N}\left(1-f_{M}(1-x)\right)=x$ and, so, $g \notin g$. In this situation we arrive at the somewhat curious conclusion that (4.1), with $\xi>1$, has no other solution than $\delta_{0}$, but that the very same equation with $\xi=1$, i.e.

$$
W \stackrel{\mathscr{L}}{=} \max _{1 \leq i \leq N} \min _{1 \leq j \leq M_{i}} W_{i, j}
$$

is always valid. 


\subsection{Connection to a fixed-point equation for weighted maxima}

In a recent article [3], Alsmeyer and Rösler studied the stochastic fixed-point equation

$$
W \stackrel{\mathcal{L}}{=} \max _{1 \leq i \leq b} T_{i} W_{i}
$$

and its min-type counterpart

$$
W \stackrel{\mathcal{L}}{=} \min _{1 \leq i \leq b} T_{i} W_{i}
$$

with $b \geq 2$ and a vector $T=\left(T_{1}, \ldots, T_{b}\right)$ of real constants. Let $\mathfrak{F}_{T}^{\max }$ and $\mathfrak{F}_{T}^{\min }$ denote the corresponding sets of solutions in the space of distributions on $\mathbb{R}$. Since $\mathcal{L}(W) \in \mathfrak{F}_{T}^{\max }$ if and only if $\mathcal{L}(-W) \in \mathfrak{F}_{T}^{\min }$ it suffices to study only one of the two equations, here (4.2) for convenience, whereas the min-type equation was chosen in [3]. The authors provided a complete description of $\mathfrak{F}_{T}^{\min }$ in all but one case. For that exceptional case, which occurs when all $T_{i}$ are negative, they could give a necessary and sufficient condition for a distribution to be in $\mathfrak{F}_{T}^{\min }$ (see (4.3), below) but they had to leave open the question whether nontrivial solutions exist at all $\left(\mathfrak{F}_{T}^{\min } \neq\left\{\delta_{0}\right\}\right)$. Here is their result, Theorem 5.1(a) in [3], reformulated for the max-type situation.

Proposition 4.1. Let $v$ be the unique solution of the equation $v+v^{b}=1$ in $(0,1)$. A distribution $F \neq \delta_{0}$ forms a solution to (4.2) if and only if $F=v G+v^{b} \mathbb{U}_{T} G$ for some distribution $G$ on $(0, \infty)$ satisfying

$$
1-v \bar{G}(t)=\prod_{i=1}^{b}\left(1-\prod_{j=1}^{b} v \bar{G}\left(\frac{t}{T_{i} T_{j}}\right)\right)
$$

for $t \geq 0$, where $\bar{G}(t)=1-G(t)$ and the distribution $\mathbb{U}_{T} G$ on $(-\infty, 0)$ is defined by

$$
\mathbb{U}_{T} G(t):=\prod_{j=1}^{b} \bar{G}\left(\frac{t}{T_{j}}-\right), \quad t<0 .
$$

A link between our setup and (4.2) arises when observing that this equation, after one iteration, takes the form

$$
W \stackrel{\mathcal{L}}{=} \max _{1 \leq i \leq b} \min _{1 \leq j \leq b} T_{i} T_{j} W_{i, j},
$$

with i.i.d. copies $W_{i, j}$ of $W$, and that (4.4) is obviously a special case of (2.1) when $T_{1}=\cdots=$ $T_{b}:=-\xi^{1 / 2}$, in which (4.2) becomes

$$
W \stackrel{\mathcal{L}}{=} \max _{1 \leq i \leq b}-\xi^{1 / 2} W_{i}=-\xi^{1 / 2} \min _{1 \leq i \leq b} W_{i} .
$$

Thus, we have $\mathfrak{F}_{T}^{\max } \subset \mathfrak{F}_{g, \xi}$ in this case, where $g(x)=\left(1-(1-x)^{b}\right)^{b}$, and as a consequence an exhaustive description of $\mathfrak{F}_{T}^{\max }$ is now rather straightforward to obtain with the help of Theorem 2.1 and Proposition 4.1. In particular, Theorem 4.2 provides an affirmative answer to the question of whether nontrivial solutions to (4.2) exist, though for a special case only. Its proof is again deferred to Section 5. Recall from Section 1 that $\alpha$ denotes the unique fixed point of $g$ in $(0,1)$ and observe that $\alpha=1-v=v^{b}$ for $v$ as given in Proposition 4.1.

Theorem 4.2. In the situation of Proposition 4.1 further suppose that $T_{1}=\cdots=T_{b}<0$ and set $\xi:=T_{1}^{2}$. Let $g(x)=\left(1-(1-x)^{b}\right)^{b}$ and $\mathcal{F}_{+}$be defined as in Section 2 with $t_{0}=1$. Then the following assertions hold true. 
(a) If $\xi<1$, then $\mathfrak{F}_{T}^{\max }=\left\{\delta_{0}\right\}$.

(b) If $\xi=1$, then $\mathfrak{F}_{T}^{\max }=\left\{\alpha \delta_{-a}+(1-\alpha) \delta_{a}: a \geq 0\right\}$.

(c) If $\xi>1$, there is a one-to-one correspondence between $\mathfrak{F}_{T}^{\max }$ and $\mathcal{F}_{+}$in the sense that any $F \in \mathfrak{F}_{T}^{\max }$ is of the form

$$
F(t)= \begin{cases}g^{n}\left(f_{+}\left(\frac{t}{\xi^{n}}\right)\right) & \text { if } t>0, \\ \bar{F}\left(\left(\frac{-t}{\xi^{1 / 2}}\right)-\right)^{b} & \text { if } t<0, \\ \alpha & \text { if } t=0 \text { and } f_{+}(1)<1, \\ 1 & \text { if } t=0 \text { and } f_{+}(1)=1,\end{cases}
$$

for some $f_{+} \in \mathcal{F}_{+}$, where $n \in \mathbb{Z}$ is the unique integer satisfying $1 \leq t / \xi^{n}<\xi$, if $t>0$.

If $f_{+}(1)<1$ in (4.6) and thus $F \neq \delta_{0}$, we can easily check that $F=(1-\alpha) F_{+}+\alpha \mathbb{U}_{T} F_{+}$ with $F_{+}$being the normalized restriction of $F$ to the positive halfline. Defining $\mathcal{F}_{-}$as in Section 2 with $s_{0}=-1$, we further see that, in the formulation of Theorem 2.1, the distribution of $F$ as an element of $\mathfrak{F}_{g, \xi}$ corresponds to the pair $\left(f_{-}, f_{+}\right)$with $f_{-}(s):=\bar{F}\left(\left(-s / \xi^{1 / 2}\right)-\right)$ for $s \in[-\xi,-1)$. Thus, while any choice of $\left(f_{-}, f_{+}\right) \in \mathcal{F}_{-} \times \mathcal{F}_{+}$determines an element of $\mathfrak{F}_{g, \xi}$ via (2.1), only those with $f_{+} \in \mathcal{F}_{+}$and $f_{-}$, as specified above, are elements of $\mathfrak{F}_{T}^{\max }$ as well.

After these observations, let us finally turn to the question of whether the unique holomorphic solution $F^{*} \in \mathfrak{F}_{g, \xi}$ defined in Theorem 1.2 (where $\xi=g^{\prime}(\alpha)$ ) is also an element of $\mathfrak{F}_{T}^{\max }$. Rewriting the pertinent fixed-point equation (4.5) in terms of distribution functions this requires that

$$
F^{*}(t)=\left(1-F^{*}\left(\frac{-t}{\xi^{1 / 2}}\right)\right)^{b}
$$

for all $t \in \mathbb{R}$. The positive answer is given by the following result which, unlike the previous ones, will be proved in Section 6 together with Theorem 1.2.

Theorem 4.3. In the situation of Theorem 1.2, the holomorphic solution $F^{*}$ to (1.3) is also a solution to (4.7).

\section{Proofs of the results in Sections 2-4}

In order to describe $\mathfrak{F}_{g, \xi}$ for any $g \in \mathcal{g}$ and $\xi>1$, the following simple lemma elucidates the relevance of $c_{0}, c_{1} \in \mathcal{L}_{g}$.

Lemma 5.1. Let $g \in \mathcal{G}$ and $\xi>1$. Then any $F \in \mathfrak{F}_{g, \xi}$ satisfies the following assertions:

(a) $F(0-) \in\left\{0, c_{0}\right\}$ and $F(0) \in\left\{c_{1}, 1\right\}$.

(b) $F(t) \in\left[0, c_{0}\right)$ for all $t<0$ and $F(t) \in\left(c_{1}, 1\right]$ for all $t>0$.

(c) If $g(x)>x$ for $0<x<c_{0}$, then $F(t)=0$ for $t<0$.

(d) If $g(x)<x$ for $c_{1}<x<1$, then $F(t)=1$ for $t>0$.

Proof. To prove (a) and (b) first note that $F(0-)$ and $F(0) \in \mathcal{L}_{g}$ follow immediately from (2.2) by letting $t \uparrow 0$ and $t \downarrow 0$, respectively, there and using the continuity of $g$. But if 
$F(0-) \neq 0$, then $F(0-)=c_{0}$ because otherwise $F(t) \in\left[c_{0}, F(0-)\right)$ for some $t<0$ which in turn would yield the following contradiction:

$$
0=F(-\infty)=\lim _{n \rightarrow \infty} F\left(t \xi^{n}\right)=\lim _{n \rightarrow \infty} g^{n}(F(t)) \geq \lim _{n \rightarrow \infty} g\left(c_{0}\right)=c_{0} .
$$

Thus, we see that $F(0-) \in\left\{0, c_{0}\right\}$ and also that $0 \leq F(t)<c_{0}$ for all $t<0$. A similar argument shows the assertions for $F(0)$.

The proof of (c) follows from the fact that if $g(x)>x$ for $0<x<c_{0}$ then $F(t)>0$ for $t<0$ would give $F(\xi t)=g(F(t))>F(t)$, which is clearly impossible.

The proof of (d) follows from the fact that if $g(x)<x$ for $c_{1}<x<1$ then $F(t)<1$ for $t>0$ would give $F(\xi t)=g(F(t))<F(t)$, which again is impossible.

Proof of Theorem 2.1. We infer directly from the previous lemma, parts (c) and (d), that $\mathfrak{F}_{g, \xi}=\varnothing$ in case (iv), and that in cases (ii) and (iii) any solution $F \in \mathfrak{F}_{g, \xi}$ must respectively vanish on the negative and positive halfline. In what follows we restrict ourselves to the proof of case (i), as the arguments in cases (ii) and (iii) are similar. Thus, let $0<c_{0} \leq c_{1}<1$.

We check first that any $F$ of the form of (2.4) with $\left(f_{-}, f_{+}\right) \in \mathcal{F}_{-} \times \mathcal{F}_{+}$is an element of $\mathcal{F}_{g, \xi}$, that is, it satisfies the fixed-point equation (2.2) and forms a distribution function. The first property is evident by the definition of $F$. As for the second, we obtain that $F$ is right continuous and nondecreasing on each interval $\left[\xi^{n} t_{0}, \xi^{n+1} t_{0}\right), n \in \mathbb{Z}$, because it is the composition of the nondecreasing, right-continuous functions $g^{n}$ and $f_{+}$there. Now condition (2.3) ensures the asserted monotonicity of $F$ on the whole positive halfline. By a similar argument, we infer that $F$ is also nondecreasing and right continuous on $(-\infty, 0)$.

As $F$ is nondecreasing on $\mathbb{R} \backslash\{0\}$ and takes values only in $[0,1]$, we infer that

$$
q_{-\infty}:=\lim _{t \rightarrow-\infty} F(t), \quad q_{0}:=\lim _{t \downarrow 0} F(t), \quad \text { and } \quad q_{\infty}:=\lim _{t \rightarrow \infty} F(t)
$$

all exist. Moreover, by continuity of $g$,

$$
q_{\infty}=\lim _{n \rightarrow \infty} F\left(\xi^{n} t_{0}\right)=\lim _{n \rightarrow \infty} g^{n}\left(f_{+}\left(t_{0}\right)\right)=\lim _{n \rightarrow \infty} g\left(\lim _{n \rightarrow \infty} g^{n-1}\left(f_{+}\left(t_{0}\right)\right)\right)=g\left(q_{\infty}\right),
$$

i.e. $q_{\infty} \in \mathcal{L}_{g}$. As $g$ is strictly increasing and $f_{+}\left(t_{0}\right)>c_{1}$, we arrive at $q_{\infty}=1$. Analogous arguments lead to $q_{-\infty}=0$ and $q_{0}=F(0)$, so that $F \in \mathcal{F}_{g, \xi}$.

Conversely, if $F \in \mathcal{F}_{g, \xi}$, the task is to show that $\left(f_{-}, f_{+}\right):=\left(\left.F\right|_{\left[\xi s_{0}, s_{0}\right)},\left.F\right|_{\left[t_{0}, \xi t_{0}\right)}\right)$ constitutes an element of $\mathcal{F}_{-} \times \mathcal{F}_{+}$for which $F\left(\xi s_{0}\right) \in\left[0, c_{0}\right)$ and $F\left(t_{0}\right) \in\left(c_{1}, 1\right]$ must be verified. We do so for the last relation only. But $F\left(t_{0}\right) \leq c_{1}$ would imply the following contradiction:

$$
1=\lim _{n \rightarrow \infty} F\left(\xi^{n} t_{0}\right)=\lim _{n \rightarrow \infty} g^{n}\left(F\left(t_{0}\right)\right) \leq \lim _{n \rightarrow \infty} g^{n}\left(c_{1}\right)=c_{1}<1,
$$

where the monotonicity of $g$ has been utilized. This completes the proof.

Proof of Theorem 2.2(a). By assumption, we have $f_{+}\left(\xi t_{0}\right)=g\left(f_{+}\left(t_{0}\right)\right)$ and, thus, we can infer the continuity of $F$ on $\left[t_{0}, \xi t_{0}\right]$. Repeated application of (2.2) then yields the continuity of $F$ on $(0, \infty)$ since compositions of continuous functions are continuous; analogously we can deduce the continuity of $F$ on $(-\infty, 0)$. The continuity of $F$ at 0 , in fact, the left continuity of $F$ at 0 remains to be proved but can easily be deduced from the stated conditions. Details are therefore omitted. 
Proof of Theorem 2.2(b). The cases $f_{-}\left(s_{0}\right)=0$ and $f_{+}\left(t_{0}\right)=1$ are trivial and are ruled out in what follows. Suppose $f_{-}\left(s_{0}\right)>0$ and $f_{+}\left(t_{0}\right)<1$. As compositions of differentiable functions are differentiable, it suffices to check differentiability at points from the set $\left\{\xi^{n} s_{0} \mid n \in \mathbb{Z}\right\} \cup\left\{\xi^{n} t_{0} \mid n \in \mathbb{Z}\right\} \cup\{0\}$ (bearing in mind that $g^{-1}$ is differentiable on $\left(0, c_{0}\right) \cup\left(c_{1}, 1\right)$ ). For the sake of brevity we restrict ourselves to the points $\xi^{n} t_{0}$ for $n \in \mathbb{Z}$ and begin with the case in which $n=1$. The assumption that $f_{+}^{\prime}\left(\xi t_{0}\right)=\xi^{-1} g^{\prime}\left(f_{+}\left(t_{0}\right)\right) f_{+}^{\prime}\left(t_{0}\right)$ yields equality of the left-hand derivative and the right-hand derivative of $F$ in $\xi t_{0}$ and, therefore, the differentiability of $F$ in $\xi t_{0}$. By writing $F=g^{n-1} \circ F\left(\cdot / \xi^{n-1}\right)$ we get the differentiability of $F$ in $\xi^{n} t_{0}(n \in \mathbb{Z})$ and for this reason on $(0, \infty)$.

Proof of Theorem 3.1. Let $p>0, F \in \mathcal{F}_{g, \xi}$, and $W$ denote a random variable with this distribution function. Then a standard estimation together with an iterated application of (2.2) leads to

$$
\begin{aligned}
\mathrm{E}\left(W^{+}\right)^{p} & =\int_{0}^{\infty} p t^{p-1} \mathrm{P}(W>t) \mathrm{d} t \\
& \leq \mathrm{P}(W>0)+\sum_{n \geq 0}\left(\xi^{p(n+1)}-\xi^{p n}\right) \mathrm{P}\left(W>\xi^{n}\right) \\
& \leq 1+\left(\xi^{p}-1\right) \sum_{n \geq 0} \xi^{p n}\left(1-g^{n}(F(1))\right)
\end{aligned}
$$

as well as

$$
\mathrm{E}\left(W^{+}\right)^{p} \geq\left(\xi^{p}-1\right) \sum_{n \geq 0} \xi^{p n}\left(1-g^{n+1}(F(1))\right) .
$$

Similarly, for the negative part we have

$$
\left(\xi^{p}-1\right) \sum_{n \geq 0} \xi^{p n} g^{n+1}(F(-1)) \leq \mathrm{E}\left(W^{-}\right)^{p} \leq 1+\left(\xi^{p}-1\right) \sum_{n \geq 0} \xi^{p n} g^{n}(F(-1)) .
$$

The proof of (a) is as follows. If $p_{0}>0$, then $g^{\prime}(0)=\xi^{-p_{0}}<1$ and thus $g(x)<x$ on $\left(0, c_{0}\right)$. Hence, $\mathfrak{F}_{g, \xi}^{-} \neq \varnothing$ by Theorem 2.1. Now let $F \in \mathfrak{F}_{g, \xi}^{-}$and $p<p_{0}$. Then $g^{\prime}(0)<\xi^{-p}$, and we may invoke Lemma A.1(b) (with $q_{0}=0$ ) to infer, for some $\zeta \in\left(\xi^{p}, 1 / g^{\prime}(0)\right)$,

$$
\zeta^{n} g^{n}(F(-1))=\zeta^{n} F\left(\xi^{-n}\right) \rightarrow 0
$$

as $n \rightarrow \infty$, which in combination with (5.1) gives $F \in \mathbb{M}_{p}^{-}$.

The proof of (b) is as follows. If $p>p_{0}$, then $g^{\prime}(0)>\xi^{-p}$ and, by another appeal to Lemma A.1(b), we can choose $\zeta \in\left(1 / g^{\prime}(0), \xi^{p}\right)$ such that

$$
\zeta^{n} g^{n}(F(-1))=\zeta^{n} F\left(\xi^{-n}\right) \rightarrow \infty
$$

as $n \rightarrow \infty$. But this implies the lower bound for $\mathrm{E}\left(W^{-}\right)^{p}$ in (5.1) to be infinite as we can easily assess.

The proofs of (c) and (d) are analogous to the proofs of (a) and (b). Therefore, we omit further details.

Proof of Proposition 3.1. Fix any $s>0$. Since $\Psi_{F}(s)=\int_{0}^{\infty} \bar{F}\left(s^{-1} \log t\right) \mathrm{d} t$, we will study the finiteness of $I(s)=\int_{\mathrm{e}^{s}}^{\infty} \bar{F}\left(s^{-1} \log t\right) \mathrm{d} t$. By recalling $g^{k}(F(1))=F\left(\xi^{k}\right)$ and using the fact that $\log t / s=\xi^{k}$ holds if and only if $t=\mathrm{e}^{s \xi^{k}}$ for $k \in \mathbb{N}_{0}$ and $t \geq \mathrm{e}^{s}$, we infer that

$$
I(s) \leq \sum_{k \geq 0}\left(\mathrm{e}^{s \xi^{k+1}}-\mathrm{e}^{s \xi^{k}}\right) \bar{F}\left(\xi^{k}\right)=\sum_{k \geq 0}\left(\mathrm{e}^{s \xi^{k+1}}-\mathrm{e}^{s \xi^{k}}\right)\left(1-g^{k}(F(1))\right)
$$


and conversely that

$$
I(s) \geq \sum_{k \geq 0}\left(\mathrm{e}^{s \xi^{k+1}}-\mathrm{e}^{s \xi^{k}}\right) \bar{F}\left(\xi^{k+1}\right)=\sum_{k \geq 0}\left(\mathrm{e}^{s \xi^{k+1}}-\mathrm{e}^{s \xi^{k}}\right)\left(1-g^{k+1}(F(1))\right) .
$$

This yields assertion (3.1) for positive $s$ because $\mathrm{e}^{s \xi^{k+1}}-\mathrm{e}^{s \xi^{k}}=\mathrm{e}^{s \xi^{k+1}}\left(1-\left(\mathrm{e}^{-s \xi^{k}}\right)^{\xi-1}\right) \sim$ $\mathrm{e}^{s \xi^{k+1}}$ as $k \rightarrow \infty$. The case of negative $s$ is treated similarly.

Proof of Theorem 4.1. In view of Proposition 3.1, a proof of parts (a) and (b) requires an analysis of the rate of convergence of $g^{k}(F(1))$ towards 1 and thus of the function $g$ in a left neighborhood of 1 . Parts (c) and (d) are then obtained in a similar manner by an analysis of the rate of convergence of $g^{k}(F(-1))$ towards 0 and thus of $g$ in a right neighborhood of 0 . This will be omitted.

We start by observing that, with $m=\min \left(m_{1}, \ldots, m_{n}\right)$,

$$
1-g(x)=1-\prod_{i=1}^{n}\left(1-(1-x)^{m_{i}}\right)=(1-x)^{m} g_{1}(x),
$$

where $g_{1}$ is a polynomial satisfying $g_{1}(1) \geq \beta:=\left|\left\{j: m_{j}=m\right\}\right|$. Consequently, for some $\varepsilon \in(0,1)$ and all $x \in[1-\varepsilon, 1]$,

$$
\frac{\beta}{2}(1-x)^{m} \leq 1-g(x) \leq 2 \beta(1-x)^{m}
$$

Fix $k_{0} \in \mathbb{N}$ so large that $1-g^{k_{0}}(F(1))<\min \left(\varepsilon,(2 \beta)^{-1}\right)$, thus $\varrho:=\log \left[2 \beta\left(1-g^{k_{0}}(F(1))\right)\right]<$ 0 . Then, for all $k>k_{0}$,

$$
\begin{aligned}
1-g^{k}(F(1)) & \leq 2 \beta\left[1-g^{k-1}(F(1))\right]^{m} \\
& \leq \cdots \leq(2 \beta)^{1+m+\cdots+m^{k-k_{0}-1}}\left[1-g^{k_{0}}(F(1))\right]^{m^{k-k_{0}}} \\
& \leq\left[2 \beta\left(1-g^{k_{0}}(F(1))\right)\right]^{m^{k-k_{0}}}
\end{aligned}
$$

and therefore

$$
\mathrm{e}^{s \xi^{k+1}}\left(1-g^{k}(F(1))\right) \leq \exp \left(m^{k}\left(s \xi\left(\frac{\xi}{m}\right)^{k}+\frac{\varrho}{m^{k_{0}}}\right)\right) .
$$

Now it is easily seen that $\sum_{k} \mathrm{e}^{s \xi^{k+1}}\left(1-g^{k}(F(1))\right)<\infty$ for all $s>0$, if $\xi<m$, and for $s$ in a right neighborhood of 0 , if $\xi=m$. By now applying Proposition 3.1, (a) is proved.

Turning to (b), we have by Lemma 5.1 that $F(1) \in\left(c_{1}, 1\right)$ if $F$ has positive mass on $(0, \infty)$. By using the left-hand inequality in (5.2), we obtain upon iteration

$$
1-g^{k}(F(1)) \geq\left(\frac{\beta}{2}\right)^{1+m+\cdots+m^{k-k_{0}-1}}\left[1-g^{k_{0}}(F(1))\right]^{m^{k-k_{0}}} \geq \exp \left(\varrho^{\prime} m^{k-k_{0}}\right)
$$

for all $k>k_{0}$ and a suitable $\varrho^{\prime}<0$. Hence, if $\xi>m$, we obtain, for each $s>0$,

$$
\liminf _{k \rightarrow \infty} \mathrm{e}^{s \xi^{k}}\left(1-g^{k}(F(1))\right) \geq \lim _{k \rightarrow \infty} \exp \left(m^{k}\left(s\left(\frac{\xi}{m}\right)^{k}+\frac{\varrho^{\prime}}{m^{k_{0}}}\right)\right)=\infty,
$$

and thus, by applying Proposition 3.1 again, $\Psi_{F}(s)=\infty$. 
Proof of Theorem 4.2. Recall that here $T_{1}=\cdots=T_{b}<0, g(x)=\left(1-(1-x)^{b}\right)^{b}$, $\xi=T_{1}^{2}$, and that $\mathfrak{F}_{T}^{\max } \subset \mathfrak{F}_{g, \xi}$. It is not difficult to check, for all three cases, that any $F$ of the asserted form is indeed an element of $\mathfrak{F}_{T}^{\max }$. Therefore, we only prove that this form is also necessary.

The proof of (a) is as follows. If $F \in \mathfrak{F}_{T}^{\max }$ and $\xi<1$, then

$$
F(t)=g^{n}\left(F\left(\frac{t}{\xi^{n}}\right)\right) \begin{cases}\geq F\left(\frac{t}{\xi^{n}}\right) \rightarrow 1 & \text { if } t>0, \\ \leq F\left(\frac{t}{\xi^{n}}\right) \rightarrow 0 & \text { if } t<0, \quad \text { as } n \rightarrow \infty,\end{cases}
$$

implying $F=\delta_{0}$.

The proof of (b) is as follows. If $F \in \mathfrak{F}_{T}^{\max } \backslash\left\{\delta_{0}\right\}$ and $\xi=1$, then $F(t) \in \mathcal{L}_{g}=\{0, \alpha, 1\}$ for all $t \in \mathbb{R}$ and $F(0)=\alpha$. Consequently, $F=\alpha \delta_{a^{\prime}}+(1-\alpha) \delta_{a}$ for suitable $a^{\prime} \leq 0<a$. Now use (4.5) with $\xi=1$ to infer that (with $W \stackrel{\mathcal{L}}{=} F$ )

$$
\mathrm{P}(W=x)=\mathrm{P}\left(\min _{1 \leq i \leq b} W_{i}=-x\right)
$$

for each $x$ and thus $a^{\prime}=-a$.

The proof of (c) is as follows. If $F \in \mathfrak{F}_{T}^{\max }$ and $\xi>1$, then $F$ is also an element of $\mathfrak{F}_{g, \xi}$ whence, by Theorem 2.1 (case (i)), there exists $f_{+} \in \mathcal{F}_{+}$such that $F$ is of the asserted form for $t \geq 0$. But $F(t)$ for $t<0$ is then necessarily of the asserted form by Proposition 4.1.

\section{Proofs of Theorem 1.2 and Theorem 4.3}

Throughout this section we always assume that $g(x)=\left(1-(1-x)^{b}\right)^{b}$ for some $b \geq 2$ with unique fixed point $\alpha \in(0,1)$. Recall from Section 1 that $\xi=g^{\prime}(\alpha)>1$ and that $\left(a_{n}\right)_{n \geq 0}$ is recursively defined by $a_{0}=\alpha, a_{1}=1$, and (see (1.6))

$$
a_{n}=\frac{1}{\xi^{n}-\xi} \sum_{k=2}^{b^{2}} c_{k} \sum_{\substack{j_{1}+\cdots+j_{k}=n, j_{1} \geq 1, \ldots, j_{k} \geq 1}} a_{j_{1}} \cdots a_{j_{k}}
$$

for $n \geq 2$, where $c_{0}, \ldots, c_{b^{2}}$ are the coefficients of $g$ in its polynomial expansion about $\alpha$. Finally, let $F^{*}$ be the limiting distribution function given in Theorem 1.1 by which we already know that $F^{*}$ is continuous and satisfying $0<F^{*}<1, F^{*}(0)=\alpha$, and (1.3).

Let $\delta_{g, \xi}$ be the operator on the set of distribution functions on $\mathbb{R}$ which maps $F$ to $g(F(\cdot / \xi))$. Then $F$ is a fixed point for $\S_{g, \xi}$, i.e. $\wp_{g, \xi}(F)=F$, if and only if $F$ solves (1.3). Furthermore, the convergence statement of Theorem 1.1 may then be restated as

$$
\lim _{n \rightarrow \infty} \oint_{g, \xi}^{n}\left(F_{\alpha}\right)=F^{*},
$$

with $F_{\alpha}$ being the uniform distribution on $(-\alpha, 1-\alpha)$ and the limit being taken in the sense of weak convergence. Crucial for our proof of Theorem 1.2 below is the following extension of (6.1) on the behavior of $g_{g, \xi}^{n}(F)$ for distribution functions $F$ having a left- and a right-hand derivative at 0 . 
Proposition 6.1. Let $F$ be any distribution function with $F(0)=\alpha$ and with left- and righthand derivatives $c_{-}$and $c_{+}$, respectively, at 0 . Then $\delta_{g, \xi}^{n}(F)$ converges weakly to a limiting distribution function $G$ if and only if $c_{-}$and $c_{+}$are both positive, in which case

$$
G(t)=F^{*}\left(c_{-} t\right) \mathbf{1}_{(-\infty, 0)}(t)+F^{*}\left(c_{+} t\right) \mathbf{1}_{[0, \infty)}(t), \quad t \in \mathbb{R}
$$

Note that, if $W^{*}$ denotes a random variable with distribution function $F^{*}$, then $G$ is the distribution function of $c_{+}^{-1}\left(W^{*}\right)^{+}-c_{-}^{-1}\left(W^{*}\right)^{-}$.

Proof. Set $G_{n}:=8_{g, \xi}^{n}(F)$ with $F$ as stated. Since $G$ is continuous we must prove that $G_{n}(t) \rightarrow G(t)$ for all $t \in \mathbb{R}$ and must do this for the cases in which $t<0$ and $t \geq 0$ separately. However, we will only consider the case in which $t \geq 0$ because the arguments in the other case are analogous.

By assumption on $F$,

$$
F(s)=\alpha+c_{+} s+r(s)
$$

for all $s \geq 0$, where $s^{-1} r(s) \rightarrow 0$ as $s \downarrow 0$. Fix $\varepsilon>0$ and then $\delta>0$ small enough so that $r(s) \leq \varepsilon s$ for $s \in[0, \delta]$. It follows, for all $n, t$ such that $t / \xi^{n} \leq \delta$ and $\left(c_{+}+\varepsilon\right) t / \xi^{n} \leq 1-\alpha$, that

$$
G_{n}(t)=g^{n}\left(F\left(\frac{t}{\xi^{n}}\right)\right) \leq g^{n}\left(\alpha+\frac{\left(c_{+}+\varepsilon\right) t}{\xi^{n}}\right)=g^{n}\left(F_{\alpha}\left(\frac{\left(c_{+}+\varepsilon\right) t}{\xi^{n}}\right)\right)
$$

and, then, by applying Theorem 1.1 (or (6.1)) it follows that

$$
\limsup _{n \rightarrow \infty} G_{n}(t) \leq F^{*}\left(\left(c_{+}+\varepsilon\right) t\right) \quad \text { for all } t \geq 0 .
$$

But $\varepsilon>0$ was chosen arbitrarily and $F^{*}$ is continuous, whence (6.2) persists to hold with $\varepsilon=0$, that is

$$
\limsup _{n \rightarrow \infty} G_{n}(t) \leq F^{*}\left(c_{+} t\right) \quad \text { for all } t \geq 0
$$

Now, if $c_{+}=0$ then $F^{*}(0)=\alpha<1$ shows that $G_{n}$ cannot converge to a proper distribution function. So, $c_{+}>0$ is necessary for $G_{n} \rightarrow G$ and supposed hereafter. Fix any $\varepsilon \in\left(0, c_{+}\right)$ and then $\delta>0$ such that $r(s) \geq-\varepsilon s$ for $s \in[0, \delta]$. It follows, for all $n, t$ such that $t / \xi^{n} \leq \delta$ and $\left(c_{+}-\varepsilon\right) t / \xi^{n} \leq 1-\alpha$, that

$$
G_{n}(t)=g^{n}\left(F\left(\frac{t}{\xi^{n}}\right)\right) \geq g^{n}\left(\alpha+\frac{\left(c_{+}-\varepsilon\right) t}{\xi^{n}}\right)=g^{n}\left(F_{\alpha}\left(\frac{\left(c_{+}-\varepsilon\right) t}{\xi^{n}}\right)\right) .
$$

Hence, by applying Theorem 1.1 again and upon letting $\varepsilon$ tend to 0 , we infer that

$$
\liminf _{n \rightarrow \infty} G_{n}(t) \geq F^{*}\left(c_{+} t\right) \quad \text { for all } t \geq 0,
$$

which in combination with (6.3) yields $G_{n}(t) \rightarrow G(t)$ for $t \geq 0$.

Proof of Theorem 1.2. The first step is to show that $H(z):=\sum_{n \geq 0} a_{n} z^{n}, z \in \mathbb{C}$, defines an entire function satisfying $H(z)=g(H(z / \xi))$. In a second step we must verify that its restriction to the real line constitutes a distribution function and thus a $\mathcal{C}^{\infty}$-solution to (1.3). Finally, the uniqueness of $H$ as a solution to (1.3) satisfying $H(0)=\alpha$ and $H^{\prime}(0)=1$ must be shown, thus giving $H=F^{*}$. 
Set $a_{n}^{(\gamma)}:=a_{n} \gamma^{n}$ for $n \in \mathbb{N}_{0}$ and $\gamma>0$. Note that $\left(a_{n}^{(\gamma)}\right)_{n \geq 0}$ satisfies the same recursion as $\left(a_{n}\right)_{n \geq 0}$, but with initial values $a_{0}^{(\gamma)}=\alpha$ and $a_{1}^{(\gamma)}=\gamma$. For $n \geq 2$, we have

$$
\begin{aligned}
\left|a_{n}^{(\gamma)}\right| & =\frac{1}{\xi^{n}-\xi}\left|\sum_{k=2}^{b^{2}} c_{k} \sum_{j_{1}+\cdots+j_{k}=n} a_{j_{1}}^{(\gamma)} \cdots a_{j_{k}}^{(\gamma)}\right| \\
& \leq \frac{1}{\xi^{n}-\xi} \sum_{k=2}^{b^{2}}\left|c_{k}\right|\left(\begin{array}{l}
n-1 \\
k-1
\end{array}\right) \max \left\{\left|a_{1}^{(\gamma)}\right|, \ldots,\left|a_{n-1}^{(\gamma)}\right|\right\}^{k} \\
& \leq \frac{1}{\xi^{n}-\xi} \max \left\{1,\left|a_{1}^{(\gamma)}\right|, \ldots,\left|a_{n-1}^{(\gamma)}\right|\right\}^{b^{2}} p(n),
\end{aligned}
$$

where $p(n):=\sum_{k=2}^{b^{2}}\left|c_{k}\right|\left(\begin{array}{c}n-1 \\ k-1\end{array}\right)$ is a polynomial in $n \in \mathbb{N}$. Choose $n_{0} \in \mathbb{N}$ so large that $p(n) \leq \xi^{n}-\xi$ for all $n \geq n_{0}$ and then fix $\gamma \in(0,1)$ small enough such that

$$
\max \left\{\left|a_{1}^{(\gamma)}\right|, \ldots,\left|a_{n_{0}-1}^{(\gamma)}\right|\right\} \leq 1 .
$$

Then (6.4) obviously implies that $\left|a_{n}^{(\gamma)}\right| \leq 1$ for all $n \geq 0$ and thus that the power series $\sum_{n \geq 0} a_{n}^{(\gamma)} z^{n}$ converges in the unit disk $\{z \in \mathbb{C}:|z|<1\}$. As $a_{n}=a_{n}^{(\gamma)} \gamma^{-n}$ for all $n \geq 0$, this is equivalent to $H(z)$ being well-defined as a convergent power series in $\{z \in \mathbb{C}:|z|<\gamma\}$. From the recursion, (1.6), in combination with $c_{1}=g^{\prime}(\alpha)=\xi$, we infer, for $n \geq 2$, that

$$
a_{n}=\frac{1}{\xi^{n}-\xi} \sum_{k=1}^{b^{2}} c_{k} \sum_{j_{1}+\cdots+j_{k}=n} a_{j_{1}} \cdots a_{j_{k}}-\frac{\xi}{\xi^{n}-\xi} a_{n},
$$

and thereupon that

$$
a_{n}=\xi^{-n} \sum_{k=1}^{b^{2}} c_{k} \sum_{j_{1}+\cdots+j_{k}=n} a_{j_{1}} \cdots a_{j_{k}} .
$$

Consequently, we have, for $|z|<\gamma$,

$$
\begin{aligned}
H(z)-\alpha=\sum_{n \geq 1} a_{n} z^{n} & =\sum_{n \geq 1} \sum_{k=1}^{b^{2}} c_{k} \sum_{j_{1}+\cdots+j_{k}=n} a_{j_{1}} \cdots a_{j_{k}}\left(\frac{z}{\xi}\right)^{n} \\
& =\sum_{k=1}^{b^{2}} c_{k} \sum_{j_{1} \geq 1} \cdots \sum_{j_{k} \geq 1} a_{j_{1}}\left(\frac{z}{\xi}\right)^{j_{1}} \cdots a_{j_{k}}\left(\frac{z}{\xi}\right)^{j_{k}} \\
& =\sum_{k=1}^{b^{2}} c_{k}\left(H\left(\frac{z}{\xi}\right)-\alpha\right)^{k} \\
& =g\left(H\left(\frac{z}{\xi}\right)\right)-\alpha
\end{aligned}
$$

that is $H(z)=g(H(z / \xi))$ for $|z|<\gamma$. But this functional equation yields, upon iteration, that, as claimed, $H$ extends to a holomorphic function in the entire complex plane satisfying the very equation everywhere. 
Since $H(0)=\alpha \in(0,1)$ and $H^{\prime}(0)=1$ we have that $H$ defines a strictly increasing $\mathrm{C}^{\infty}$-function in $(-\varepsilon, \varepsilon)$ for some $\varepsilon>0$. Fix any $s_{0}<0<t_{0}$ such that $\left[\xi s_{0}, s_{0}\right] \cup\left[t_{0}, \xi t_{0}\right] \subset$ $(-\varepsilon, \varepsilon)$ as well as $0<H\left(s_{0}\right)<\alpha<H\left(t_{0}\right)<1$. Let $f_{-}$and $f_{+}$denote the restrictions of $H$ to $\left[\xi s_{0}, s_{0}\right]$ and $\left[t_{0}, \xi t_{0}\right]$, respectively. Then $\left(f_{-}, f_{+}\right) \in \mathcal{F}_{-} \times \mathcal{F}_{+}$with $\mathcal{F}_{-}$and $\mathcal{F}_{+}$as defined in Section 2. Hence, by Theorem 2.1, this pair defines a distribution function via (2.4) which forms a solution to (1.3). But this function is clearly the function $H$ restricted to the real line because this restriction also satisfies (1.3) as stated above. We have thus completed the second step of the proof.

In order to conclude $H=F^{*}$ on $\mathbb{R}$, we may invoke Proposition 6.1 with $F=H$ and thus $c_{-}=c_{+}=1$ to infer that

$$
H=g_{g, \xi}^{n}(H) \rightarrow F^{*}, \quad n \rightarrow \infty
$$

which gives the result.

Finally, we must argue that $\Psi_{F^{*}}(t)$ is finite for all $t \in \mathbb{R}$. An elementary but tedious calculation carried out in [5] showed that $\xi=g^{\prime}(\alpha)<b$. Hence, we arrive at the desired conclusion by an appeal to Theorem 4.1(a) and (c) with $n=m_{1}=\cdots=m_{n}=b \geq 2$.

Proof of Theorem 4.3. Defining $\hat{g}(x):=(1-x)^{b}$ and

$$
\delta_{\hat{g}, \xi^{1 / 2}}(F)(t):=\left(1-F\left(\frac{-t}{\xi^{1 / 2}}\right)-\right)^{b}
$$

for distribution functions $F$, we must obviously prove that $\delta_{\hat{g}, \xi^{1 / 2}}\left(F^{*}\right)=F^{*}$. Now we use the facts that $\wp_{g, \xi}=\wp_{\hat{g}, \xi^{1 / 2}}^{2}$ and $\wp_{g, \xi}\left(F^{*}\right)=F^{*}$ to infer that

$$
8_{\hat{g}, \xi^{1 / 2}}^{2 n+1}\left(F^{*}\right)=8_{\hat{g}, \xi^{1 / 2}}\left(8_{g, \xi}^{n}\left(F^{*}\right)\right)=\S_{\hat{g}, \xi^{1 / 2}}\left(F^{*}\right)
$$

for all $n \geq 0$. On the other hand, Proposition 6.1 implies that

$$
\S_{\hat{g}, \xi^{1 / 2}}^{2 n+1}\left(F^{*}\right)=\wp_{g, \xi}^{n}\left(\wp_{\hat{g}, \xi^{1 / 2}}\left(F^{*}\right)\right) \rightarrow F^{*} \quad(\text { weakly })
$$

as $n \rightarrow \infty$, if we can verify that $G:=\S_{\hat{g}, \xi^{1 / 2}}\left(F^{*}\right)$ is a distribution function having $G(0)=\alpha$ and derivative 1 at 0 . Together with (6.5) this clearly gives the desired conclusion.

To prove $G^{\prime}(0)=1$ for $G(t)=\left(1-F^{*}\left(-t / \xi^{1 / 2}\right)\right)^{b}$ (no left-hand limit is needed here as $F^{*}$ is continuous), we note that

$$
G^{\prime}(t)=\frac{b}{\xi^{1 / 2}} F^{*^{\prime}}\left(\frac{-t}{\xi^{1 / 2}}\right)\left(1-F^{*}\left(\frac{-t}{\xi^{1 / 2}}\right)\right)^{b-1},
$$

and, thus, obtain $G^{\prime}(0)=b(1-\alpha)^{b-1} / \xi^{1 / 2}$, as $F^{*}(0)=\alpha$ and $F^{*^{\prime}}(0)=1$. So, it remains to verify that $\xi^{1 / 2}=b(1-\alpha)^{b-1}$. But recalling that $v=1-\alpha$ solves the equation $v+v^{b}=1$ this follows from

$$
\xi=g^{\prime}(\alpha)=b^{2}(1-\alpha)^{b-1}\left(1-(1-\alpha)^{b}\right)^{b-1}=b^{2}(1-\alpha)^{2 b-2} .
$$

Finally, noting that $G(0)=\left(1-F^{*}(0)\right)^{b}=(1-\alpha)^{b}=\alpha$ the proof is complete. 


\section{Concluding remarks}

We have seen that the maximin fixed point equations studied in this article are closely related to the iterations of certain strictly increasing continuous functions $g:[0,1] \rightarrow[0,1]$ with $g(0)=0$ and $g(1)=1$. In fact, $g$ is simply a polynomial if the number of involved maxima and minima in the pertinent maximin equation is not random as is the case in our main application of game tree evaluation described in the introduction. We have chosen the class $g$ of functions $g$ so as to meet two competing goals. On the one hand, we wanted to cover a relatively wide class of maximin equations, including (1.4) and (2.1), and to study them within a unified framework. On the other hand, we wanted to keep technicalities as well as the number of cases to be discussed separately at a comprehensive level. A thorough analysis of the equation $F(t)=g(F(t / \xi))$ for general increasing and continuous functions $g:[0,1] \rightarrow[0,1]$ with $g(0)=0$ and $g(1)=1$, in which case $\mathcal{L}_{g} \backslash\{0,1\}$ could be either a void or a finite or infinite subset of $(0,1)$, would have required various partly tedious discussions of extra cases without gaining much additional insight. We should mention, however, that this would have included the pure max-type and min-type equations

$$
W \stackrel{\mathcal{L}}{=} \xi \max _{1 \leq i \leq b} W_{i} \quad \text { and } \quad W \stackrel{\mathcal{L}}{=} \xi \min _{1 \leq i \leq b} W_{i}, \quad \xi>1, b \in \mathbb{N},
$$

pertaining to $g(x)=x^{b}$ and $g(x)=1-(1-x)^{b}$, respectively, neither of which have any fixed points between 0 and 1 . For such $g$ that are either strictly smaller or larger, respectively, than the identity function on $(0,1)$, a one-to-one correspondence between $\mathfrak{F}_{g, \xi}$ and $\mathcal{F}_{-}$, respectively $\mathcal{F}_{+}$can be shown fairly easily by similar arguments as in the proof of Theorem 2.1 .

We have shown that the maximin equation (2.1) with $n=m_{1}=\cdots=m_{n}=b \geq 2$ yields upon one iteration of the pure min-type equation (4.5) with negative scaling factor $-\xi^{1 / 2}$. We have further shown that the holomorphic solution $F^{*}$ of (2.1) in that case also solves (4.5), and thus (4.7) in terms of distribution functions (Theorem 4.3). As

$$
F^{*}(t)=\sum_{n \geq 0} a_{n} x^{n}
$$

with $a_{0}=1, a_{1}=\alpha$ (Theorem 1.2), the latter equation takes the form

$$
\sum_{n \geq 0} a_{n} x^{n}=\left(1-\sum_{n \geq 0} a_{n} \frac{(-1)^{n}}{\xi^{n / 2}} x^{n}\right)^{b},
$$

which may be used to provide a simplified recursion for the $a_{n}$, as opposed to using (1.6), via a comparison of coefficients of the power series on the left- and right-hand side of (7.1). This has been done by the second author in [5], the result being

$$
a_{n}=\frac{(-1)^{n} \xi^{-n / 2}}{1+(-1)^{n} \xi^{-(n-1) / 2}}\left(\sum_{k=2}^{b} \hat{c}_{k} \sum_{\substack{j_{1}+\ldots+j_{k}=n, j_{1} \geq 1, \ldots, j_{k} \geq 1}} a_{j_{1}} \cdots a_{j_{k}}\right),
$$

where $\hat{c}_{0}, \ldots, \hat{c}_{b}$ are the coefficients of $\hat{g}(x)=(1-x)^{b}$ in its expansion about $\alpha$. Note that the sum in this recursion involves only $b-1$ terms while that in (1.6) has $b^{2}-1$ terms. 


\section{Appendix A. Fixed points of real functions on the unit interval}

The following simple lemma collects some basic convergence properties of the functions $g$ considered in this article and is stated without proof.

Lemma A.1. Let $g:[0,1] \rightarrow \mathbb{R}$ be nondecreasing and continuously differentiable and furthermore let $0 \leq q_{0}<q_{1} \leq 1$ be two fixed points of $g$ such that $g(x) \neq x$ for $q_{0}<x<q_{1}$.

(a) If $g(x)>x$ on $\left(q_{0}, q_{1}\right)$ then

$$
\lim _{n \rightarrow \infty} \sup _{q \leq x \leq q_{1}} \gamma^{-n}\left|q_{1}-g^{n}(x)\right|=0
$$

for each $\gamma>g^{\prime}\left(q_{1}\right)$ and $q \in\left(q_{0}, q_{1}\right]$. If $g^{\prime}\left(q_{1}\right)>0$ then, furthermore,

$$
\lim _{n \rightarrow \infty} \inf _{q_{0} \leq x \leq q} \beta^{-n}\left|q_{1}-g^{n}(x)\right|=\infty
$$

for each $0<\beta<g^{\prime}\left(q_{1}\right)$ and $q \in\left[q_{0}, q_{1}\right)$.

(b) If $g(x)<x$ on $\left(q_{0}, q_{1}\right)$ then

$$
\lim _{n \rightarrow \infty} \sup _{q_{0} \leq x \leq q} \gamma^{-n}\left|q_{0}-g^{n}(x)\right|=0
$$

for each $\gamma>g^{\prime}\left(q_{0}\right)$ and $q \in\left(q_{0}, q_{1}\right]$. If $g^{\prime}\left(q_{0}\right)>0$ then, furthermore,

$$
\lim _{n \rightarrow \infty} \inf _{q \leq x \leq q_{1}} \beta^{-n}\left|q_{1}-g^{n}(x)\right|=\infty
$$

for each $0<\beta<g^{\prime}\left(q_{1}\right)$ and $q \in\left[q_{0}, q_{1}\right)$.

Our second lemma shows that the function $g$ pertaining to the maximin equation (2.1) has exactly one fixed point in $(0,1)$.

Lemma A.2. Given any $n, m_{1}, \ldots, m_{n} \geq 2$, the polynomial $g(x)=\prod_{i=1}^{n}\left(1-(1-x)^{m_{i}}\right)$ has exactly one fixed point in $(0,1)$ and further satisfies $g^{\prime}(0)=g^{\prime}(1)=0$.

Proof. Without loss of generality let $m_{1} \leq m_{2} \leq \cdots \leq m_{n}$. It is immediate from

$$
g^{\prime}(x)=\sum_{i=1}^{n}\left(m_{i}(1-x)^{m_{i}-1} \prod_{j \neq i}\left(1-(1-x)^{m_{j}}\right)\right)
$$

that $g^{\prime}(0)=g^{\prime}(1)=0$ because $n, m_{1}, \ldots, m_{n}>1$. As a consequence, there must be at least one $c \in(0,1) \cap \mathcal{L}_{g}$. After some elementary algebra we find that $g(1-x)=1-x$ for $x \in(0,1)$ holds if and only if

$$
\sum_{k=0}^{m_{1}-1} x^{k}=\frac{1-x^{m_{1}}}{1-x}=\prod_{j=2}^{n} \frac{1}{1-x^{m_{j}}}=\sum_{k_{2}, \ldots, k_{n} \geq 0} x^{k_{2} m_{2}+\cdots+k_{n} m_{n}} .
$$

Now observe that the left-hand sum constitutes the generating function of the counting measure $\lambda_{1}$, say, on $\left\{0, \ldots, m_{1}-1\right\}$, while the right-hand sum is the generating function of an infinite measure $\lambda_{2}$ on $\mathbb{N}_{0}$ which puts mass 1 at 0 but no mass on $\left\{1, \ldots, m_{1}-1\right\}$ as $m_{1} \leq m_{2} \leq$ $\cdots \leq m_{n}$. As a consequence, the signed measure $\lambda_{1}-\lambda_{2}$ has 0 mass at 0 , mass 1 at each 
$k=1, \ldots, m_{1}-1$, and either mass 0 or nonpositive mass less than or equal to -1 at each $k \geq m_{1}$ (the latter occurring infinitely often). This shows that $\Delta(n):=\left(\lambda_{1}-\lambda_{2}\right)(\{0, \ldots, n\}), n \geq 0$, is unimodal with $\Delta(0)=0, \Delta(1)=1$, and $\lim _{n \rightarrow \infty} \Delta(n)=-\infty$. We will now argue that

$$
\sum_{k=0}^{m_{1}-1} x^{k}-\sum_{k_{2}, \ldots, k_{n} \geq 0} x^{k_{2} m_{2}+\cdots+k_{n} m_{n}}=(1-x) \sum_{n \geq 1} \Delta(n) x^{n}
$$

has a unique root $x^{*}$ in $(0,1)$ so that $1-x^{*}$ constitutes the unique fixed point of $g$ in $(0,1)$. Define

$$
H(x):=\sum_{n \geq 1} \Delta(n) x^{n}=\sum_{n=1}^{m^{*}-1}|\Delta(n)| x^{n}-\sum_{n \geq m^{*}}|\Delta(n)| x^{n}, \quad x \in[0,1),
$$

where $m^{*}:=\inf \{n: \Delta(n)<0\} \geq m_{1} \geq 2$, and note that

$$
x H^{\prime}(x)-m^{*} H(x)=\sum_{n=1}^{m^{*}-1}\left(n-m^{*}\right)|\Delta(n)| x^{n}-\sum_{n>m^{*}}\left(n-m^{*}\right)|\Delta(n)| x^{n} \leq-x
$$

for $x \in[0,1)$. Since $H(0)=0, H^{\prime}(0)=\Delta(1)=1$, and $\lim _{x \uparrow 1} H(x)=-\infty$, there is at least one root $x^{*}$ in $(0,1)$. By the previous inequality, we also have $H^{\prime}\left(x^{*}\right) \leq-1$ and thus $H(x)<0$ in a right neighborhood of $x^{*}$. But if $H(x)<0$ for $x \in(0,1)$, then $H^{\prime}(x) \leq x^{-1} m^{*} H(x)<0$, and this implies that $H(x)<0$ for all $x \in\left(x^{*}, 1\right)$ and thus the asserted uniqueness of $x^{*}$.

\section{References}

[1] Aldous, D. and Bandhyopadhyay, A. (2005). A survey of max-type recursive distributional equations. Ann. Appl. Probab. 15, 1047-1110.

[2] Ali Khan, T., Devroye, L. And Neininger, R. (2005). A limit law for the root value of minimax trees. Electron. Commun. Prob. 10, 273-281.

[3] Alsmeyer, G. And Rösler, U. (2007). A stochastic fixed-point equation for weighted minima and maxima. To appear in Ann. Inst. H. Poincaré Prob. Statist.

[4] Jagers, P. AND Rösler, U. (2004). Stochastic fixed points for the maximum. In Mathematics and Computer Science, III, eds M. Drmota et al. , Birkhäuser, Basel, pp. 325-338.

[5] MeIners, M. (2006). Über stochastische Maximin-Fixpunktgleichungen. Diploma Thesis, University of Münster.

[6] NeINinger, R. AND Rüschendorf, L. (2005). Analysis of algorithms by the contraction method: additive and max-recursive sequences. In Interacting Stochastic Systems, eds J. D. Deuschel and A. Greven, Springer, Heidelberg, pp. 435-450.

[7] Pearl, J. (1980). Asymptotic properties of minimax trees in game-searching procedures. Artificial Intelligence 14, 113-138. 